\title{
Tromboembolia pulmonar en el embarazo y puerperio
}

\author{
Antonio Gerardo Rojas-Sánchez, ${ }^{*} \bowtie$ Gabriela Navarro-de la Rosa, ${ }^{*}$ Julio César Mijangos-Méndez, ${ }^{\S}$ \\ Ricardo Campos-Cerda ${ }^{I I}$
}

*Jefe del Servicio de Fisiología Pulmonar del Hospital Civil de Guadalajara "Fray Antonio Alcalde”, Guadalajara, Jalisco;

"Depto. de Nefrología, Hospital General Regional Núm. 45, IMSS, Guadalajara, Jalisco;

§UTI, Hospital Civil de Guadalajara "Fray Antonio Alcalde", Guadalajara, Jalisco;

"UCI, Hospital General Regional Núm. 46, IMSS, Guadalajara, Jalisco. México.

Trabajo recibido: 10-IV-2013; aceptado: 05-IX-2013

\begin{abstract}
RESUMEN. La mujer embarazada tiene cinco veces el riesgo de desarrollar tromboembolia pulmonar (TEP) con respecto a las no embarazadas. Los cambios cardiovasculares y de la coagulación propios del embarazo pueden facilitar los eventos de TEP. El riesgo se incrementa en la presencia de otros factores: edad, multiparidad, lupus eritematoso sistémico y TEP previa. El proceso diagnóstico inicia con la sospecha clínica. La determinación de dímeros-D es útil principalmente por su valor predictivo negativo. La angiotomografía pulmonar helicoidal tiene una alta sensibilidad y especificidad en el diagnóstico, mientras que la arteriografía pulmonar sigue siendo el estándar de oro para el diagnóstico definitivo. El tratamiento de la TEP consiste en la anticoagulación tan pronto se establezca el diagnóstico. Tanto la heparina no fraccionada como la de bajo peso molecular son los medicamentos de elección sobre los antagonistas de la vitamina K, principalmente debido a que las heparinas no atraviesan la barrera útero-placentaria, presentan menos efectos adversos y tienen una vida media más corta.
\end{abstract}

Palabras clave: Tromboembolismo pulmonar, embarazo, puerperio, enfermedad tromboembólica venosa, trombosis venosa profunda.

ABSTRACT. The pregnant woman has five times greater the risk of developing pulmonary thromboembolism with respect to non-pregnant, mainly due to the cardiovascular and coagulation changes characteristic of pregnancy, and the risk increases with the presence of other factors such as age, previous pulmonary embolism, systemic lupus erythematosus and multiparity, among others. The diagnostic process begins with clinical suspicion. Determination of $\mathrm{D}$-dimer is useful because its negative predictive value. The helical computed tomography has a high sensitivity and specificity in the diagnosis of pulmonary thromboembolism, while pulmonary angiography remains the definitive diagnostic study. Treatment consists of anticoagulation as soon as the diagnosis is established, both unfractionated heparin and low molecular weight heparins are the drugs of choice over vitamin $\mathrm{K}$ antagonists, primarily because the first ones do not cross the placental barrier, and have fewer side effects due to their short half-life.

Key words: Pulmonary thromboembolism, pregnancy, puerperium, venous thromboembolic disease, deep vein thrombosis.

\section{INTRODUCCIÓN}

Como evento natural, el embarazo debería estar libre de complicaciones y, por lo tanto, de muerte. Los fallecimientos asociados a la gestación, parto, puerperio y sus procesos de atención se consideran, en su mayoría, evitables con los recursos de la medicina actual. Sin embargo, cada año fallecen poco más de medio millón de mujeres en todo el mundo por complicaciones obstétricas. ${ }^{1}$ Las principales causas informadas de muerte materna en países desarrollados son: la enfermedad hipertensiva asociada al embarazo (16.1\%), la tromboembolia pulmonar (TEP) $(14.9 \%)$ y la hemorragia obstétrica (13.4\%). ${ }^{2}$

El embarazo es un estado fisiológico con alto riesgo reconocido para el desarrollo de complicaciones tromboembólicas. Durante la gestación, parto y puerperio, la mujer embarazada tiene factores de riesgo aumentados para el desarrollo de TEP. La incidencia para TEP en mujeres embarazadas comparadas con las que no lo están es de cuatro a cinco veces mayor ( 0.76 a 1.72/1,000 embarazos). En Estados Unidos este padecimiento es una de las principales causas de muerte materna, y es responsable del $11 \%$ de ellas. En México, la incidencia de TEP es de 4.7 casos por 10,000 consultas obstétricas, y la mortalidad reportada por esta causa es de $6.6 \% .^{3-5}$

Debido a la importancia que tiene el desarrollo de TEP en la mujer embarazada, así como la falta de estudios donde incluir a esta población de mujeres y la necesidad de un abordaje diagnóstico y tratamiento oportuno, se realizó la presente revisión bibliográfica con la intención de dar a conocer lo más relevante de la enfermedad tromboembólica pulmonar asociada al embarazo, parto y puerperio. 


\section{CAMBIOS CARDIOVASCULARES Y DE COAGULACIÓN DURANTE EL EMBARAZO}

En la mujer embarazada, durante el trabajo de parto y período posparto se presentan cambios cardiovasculares notables. El volumen sanguíneo (VS) aumenta en un $50 \%$. Estos cambios son atribuibles, en parte, a una estimulación del sistema renina-aldosterona, que es mediado por estrógenos, dando como resultado un aumento en la reabsorción de sodio y agua por el riñón. Este VS modifica el volumen sistólico y, en consecuencia, aumenta el gasto cardíaco en un 30 a 50\%. Para el tercer trimestre del embarazo, el volumen sistólico disminuye por compresión de la vena cava inferior por el útero gestante. ${ }^{3-6}$ La presión arterial sistémica (PAS) adquiere una tendencia a disminuir conforme progresa el embarazo debido al incremento de los niveles plasmáticos de prostaglandinas y del factor natriurético auricular; éstos, a su vez, condicionan una vasodilatación sistémica. Por otra parte, la disminución de la PAS también se ha asociado a la producción de calor originado por el feto..$^{5,6}$

Durante el embarazo está descrito un incremento progresivo de factores procoagulantes como el factor de Von Willebrand, V, VII, IX, X, XII y fibrinógeno, así como una resistencia adquirida al anticoagulante endógeno proteína $\mathrm{C}$ activada, y una disminución en la proteína $\mathrm{S}$, cofactor de la proteína $\mathrm{C}$. Al término del primer trimestre del embarazo ocurre estasis venosa, que se mantiene así hasta alcanzar su punto máximo en las $36-38$ semanas de gestación. Puede haber daño endotelial en los vasos pélvicos secundario a la compresión de la vena cava inferior y las venas ilíacas por el útero grávido, estasis resultante y durante el parto vaginal o la cesárea. Así, cada criterio referido por Virchow como generador de trombosis participa como riesgo para desarrollar TEP durante el embarazo y el puerperio. ${ }^{5-7}$

Durante el alumbramiento existe lesión vascular y cambios en la interfase útero-placenaria, situación que se exacerba durante el nacimiento instrumental o quirúrgico. Se activa la cascada de la coagulación, así como el consumo plaquetario de factores de coagulación y sus inhibidores, que regulan el proceso de la hemostasia. Después del nacimiento se inician las reacciones de fase aguda, que se distinguen por elevación de las concentraciones de proteína $\mathrm{C}$ reactiva y antitrombina III, principalmente durante la primera semana del puerperio. El sistema de la coagulación se normaliza entre las cuatro y seis semanas posteriores al parto. ${ }^{7-9}$

\section{FACTORES DE RIESGO}

La estasis venosa de las extremidades puede reducir la velocidad del flujo venoso hasta $50 \%$. Ésta inicia hacia la semana 25 de la gestación y se recupera en la sexta semana posterior al parto. Existe una mayor frecuencia de trombosis venosa profunda (TVP) de la extremidad inferior izquierda (80\%) debido, en parte, a la compresión de la vena ilíaca izquierda por la arteria ilíaca derecha en el sitio de su nacimiento en la aorta. El daño endotelial se ha asociado al trauma del parto por vía vaginal o cesárea, TVP previa o tabaquismo. ${ }^{10}$

Otros factores que pueden contribuir a TEP en la paciente gestante son: edad mayor a 35 años, multiparidad, TVP o TEP previa, inmovilización prolongada, inhibición de la lactancia con estrógenos, traumatismo pélvico o en las extremidades inferiores, así como la presencia de algún tipo de trombofilia hereditaria, síndrome antifosfolípido, lupus eritematoso sistémico, obesidad, enfermedades del corazón, diabetes mellitus y embarazo múltiple. ${ }^{3,4}$ Históricamente se ha reportado que el final del tercer trimestre y el posparto inmediato, es el período de mayor riesgo para la enfermedad tromboembólica venosa, y es el período del posparto inmediato el de mayor riesgo para desarrollar TEP ${ }^{4,10,11}$ (tabla 1).

\section{DIAGNÓSTICO}

El proceso diagnóstico de TEP en una mujer gestante empieza con la sospecha clínica. Muchos de los síntomas y signos son inespecíficos; el $90 \%$ de las mujeres embarazadas con TEP refieren disnea y taquipnea, y sólo el 3\% son asintomáticas. ${ }^{1,2,5}$ El diagnóstico clínico de TEP en esta población carece de precisión y de especificidad. Los signos y síntomas más frecuentes son: disnea (100\%), dolor torácico (63.3\%), taquicardia $(93.3 \%)$ y taquipnea (93.3\%). Por lo anterior, el diagnóstico de TEP durante el embarazo se realiza por combinación de probabilidad clínica y por resultados de pruebas diagnósticas objetivas. .,6,10,11 $^{-10}$

Los gases sanguíneos con frecuencia demuestran alcalosis respiratoria, hipoxemia moderada y gradiente alvéolo-arterial de oxígeno mayor a $20 \mathrm{mmHg}$. Es importante destacar los mecanismos de hiperventilación que se producen por la estimulación de la progesterona al centro respiratorio, esto se observa en la gasometría, con un discreto descenso de nivel de la $\mathrm{PaCO}_{2}$ y una consecuente disminución del nivel de $\mathrm{HCO}_{3}(3 \mathrm{mmol} / \mathrm{L})$, además, un nivel de $\mathrm{PaO}_{2}$ de 99-106 mmHg, lo que significa una alerta en aquellas pacientes con niveles de $\mathrm{PaO}_{2}$ en límites normales bajos. No obstante, la ausencia de hipoxemia no excluye la TEP. La utilidad de cuantificar los gases en la sangre arterial es tener un parámetro más en el juicio diagnóstico..$^{8-10}$

El electrocardiograma es anormal hasta en el $90 \%$ de las mujeres embarazadas con TEP; el hallazgo más 
Tabla 1. Factores de riesgo para desarrollar TEP en el embarazo.

\author{
- Edad > 35 años \\ - Obesidad \\ - Multiparidad \\ - Fumar > 10 cigarrillos al día \\ - Embarazo múltiple \\ - Reposo en cama mayor de una semana antes del parto \\ - TVP/TEP previa \\ - Preeclampsia \\ - Hemorragia posparto $>1,000 \mathrm{~mL}$ \\ - Infección posparto \\ - Daño endotelial secundario a parto o cesárea \\ — Lupus eritematoso sistémico, síndrome antifosfolípidos, enfermedad de células falciformes, enfermedad cardíaca \\ - Uso de estrógenos \\ - Deficiencia de antitrombina III \\ - Deficiencia de proteína C o S \\ — Mutación del factor $\mathrm{V}$ Leiden
}

Modificado de: Refuerzo JS, Hechtman JL, Redman ME, Whitty JE, et al. J Reprod Med 2003;48:767-770.

Abreviaturas: TEP: Tromboembolia pulmonar; TVP: Trombosis venosa profunda.

común es la taquicardia sinusal. Puede presentarse inversión inespecífica de la onda $\mathrm{T}$, depresión del segmento ST y bloqueo de rama derecha del Haz de His. El patrón electrocardiográfico $\mathrm{S}_{1}, \mathrm{Q}_{3}, \mathrm{~T}_{3}$ es poco frecuente en TEP y embarazo; cuando se presenta es por cor pulmonale agudo asociado a hipertensión arterial pulmonar (HAP) y TEP mayor. La radiografía de tórax es normal en el $83 \%$ de los casos de TEP y embarazo, y anormal en el $17 \% .^{5,10}$ Las anormalidades más frecuentes en la radiografía de tórax son: hipertensión venocapilar $(27.5 \%)$, consolidación pulmonar (17.2\%), derrame pleural (13.7\%), oligohemia (10.3\%), opacidades $(10.3 \%)$ y atelectasia $(3.4 \%) .{ }^{5}$ El valor de la radiografía de tórax en esta población reside en poder descartar otras patologías pleuropulmonares presentes. ${ }^{5,10}$

En la mujer gestante con sospecha de TEP puede utilizarse el cuestionario de Wells para determinar la probabilidad clínica del evento; sin embargo, se requiere su validación específica en esta población de pacientes..$^{12}$

Los dímeros- $D$ tienen un papel en el diagnóstico de TEP en la población general. Su uso durante el embarazo es controversial. ${ }^{3}$ Varios estudios han confirmado su alta sensibilidad, pero baja especificidad porque pueden estar asociados a otras situaciones clínicas diversas. ${ }^{9,10}$ La determinación de dímeros-D es útil en la detección temprana de anomalías de la coagulación asociadas a preeclampsia. ${ }^{13}$ Son un indicador sensible de una coagulopatía subclínica en mujeres preeclámpticas. Se han estudiado los niveles de dímeros- $D$ en las diferentes etapas del embarazo y se han encontrado en algunas gestantes sanas niveles de dímeros $D$ de hasta $875 \mathrm{ng} / \mathrm{mL}$ con preeclampsia severa 1,625 ng/mL.; con eclampsia y síndrome HELLP (hemólisis, elevación de enzimas hepáticas y plaquetas bajas) hasta $3,000 \mathrm{ng} / \mathrm{mL} .^{14,15}$

Estos resultados sugieren una elevación transitoria y progresiva de los dímeros-D en el embarazo normal, un aumento importante en casos de preeclampsia, el cual es evidentemente mayor en casos de síndrome HELLP o eclampsia. Todo esto parece explicar el hecho de que realmente existe una mayor activación del sistema de coagulación y fibrinólisis secundaria en todo embarazo normal, que se exagera en casos de hipertensión inducida por el embarazo y se relaciona progresivamente entre la gravedad de la enfermedad y los valores de dímeros-D. ${ }^{10,15}$ Por lo tanto, cuando la determinación de dímeros- $\mathrm{D}$ es normal, puede descartarse el diagnóstico de TEP (valor predictivo negativo de $97 \%$ ); empero, cuando es altamente positivo, no lo descarta (sensibilidad 96.8\%), y es necesario realizar otros estudios para confirmarlo. ${ }^{12,16}$

Otros biomarcadores que son útiles en TEP son la determinación plasmática de troponinas I, T y el péptido natriurético cerebral. Altos niveles anormales de estos biomarcadores son atribuibles al aumento de la tensión de la pared del ventrículo derecho por sobrecarga de presión con daño celular y microinfarto. Cifras de troponinas entre $0.01 \mathrm{ng} / \mathrm{mL}$ y $0.07 \mathrm{ng} / \mathrm{mL}$ se asocian con mayores defectos segmentarios y signos de disfunción del ventrículo derecho (DVD). ${ }^{17-20}$

El ecocardiograma es otro método no invasivo que identifica de forma indirecta la gravedad de la obstrucción vascular a través del grado de HAP y diferentes estadios de DVD. ${ }^{21-23}$ Es una prueba diagnóstica que puede ser útil para estratificar la gravedad de TEP en 
mujeres embarazadas, definir el grado de DVD y tomar la decisión incluso para el tratamiento de embolización y/o trombólisis, que en TEP aguda con compromiso hemodinámico y DVD está indicado. 22,24-26

La elección de la prueba diagnóstica por imagen depende de la utilidad demostrada para el diagnóstico y la exposición del feto a la radiación ionizante. Por ejemplo, el gammagrama pulmonar ventilatorio-perfusorio (V/Q) tiene una sensibilidad para el diagnóstico de TEP de $89 \%$ y una especificidad de $92 \%$, y la angiotomografía pulmonar helicoidal (ATAH) ha demostrado una sensibilidad de $83 \%$ y especificidad de $96 \%$, con un valor predictivo positivo de $96 \%$ para este diagnóstico en mujeres no embarazadas. ${ }^{5,27} \mathrm{Sin}$ embargo, la dosis de radiaciones que recibe un feto con un gammagrama pulmonar $\mathrm{V} / \mathrm{Q}$ es de $100 \mu \mathrm{Gy}$ a $370 \mu \mathrm{Gy}$, y con una ATAH, de $3.3 \mu \mathrm{Gy}$ a $130.0 \mu \mathrm{Gy}$, lo que significa que la dosis de radiación al feto es tres veces mayor con el gammagrama $V / Q$ que con la ATAH. En la mujer embarazada, la ATAH es una prueba segura para el diagnóstico de TEP. 28

La arteriografía pulmonar es considerada el estándar de oro para el diagnóstico de TEP; empero, su uso es cada vez menor debido a que requiere de equipo y personal capacitado; dicho procedimiento se reserva para un pequeño subgrupo de pacientes en quienes el diagnóstico de TEP no puede establecerse por medios menos invasivos. La exposición a radiación de este estudio es similar a la ATAH. ${ }^{10,28,29}$

En el grupo de las pacientes con TEP durante el embarazo, se carece de estudios suficientes para emitir juicios basados en la evidencia; por tanto, es necesaria mayor investigación e información en esta área. ${ }^{10} \mathrm{Ba}-$ sándonos en las pruebas diagnósticas y su seguridad en las mujeres con TEP y embarazo, proponemos un algoritmo diagnóstico, con la responsabilidad de establecer políticas de atención que brinden calidad y seguridad en la atención materno-fetal ${ }^{1,5,10}$ (figura 1).

\section{TRATAMIENTO Y PREVENCIÓN}

La paciente embarazada con diagnóstico de TEP aguda es una urgencia obstétrica cuyo tratamiento inicial está orientado al alivio de la obstrucción mediante la disolución del coágulo. La decisión de la terapia anticoagulante o fibrinolítica debe ser rápida y efectiva; requiere de un monitoreo estrecho, tanto de la madre como del producto por nacer. Es ideal tener un equipo médico multidisciplinario, incluyendo áreas de cuidados intensivos donde se puedan ofrecer medidas de soporte para las pacientes de acuerdo con la gravedad, oxígeno suplementario, ventilación mecánica, soporte hemodinámico. Es fundamental contar con una sala de hemodinamia para situaciones que requieran de inter- vencionismo urgente, como en el caso de TEP mayor con compromiso hemodinámico, que pone en riesgo la vida de la paciente y el feto. ${ }^{30,31}$

La heparina no fraccionada (HNF) es el fármaco de elección para la profilaxis y el tratamiento de la TEP durante el embarazo y el puerperio. El consenso del Colegio Americano de Médicos de Tórax (ACCP, $2012)^{11}$ señala que los antagonistas de la vitamina $K$ (AVK) deben evitarse de las semanas seis a nueve de la gestación porque pueden afectar al feto y causar embriopatía (hiperplasia del tercio medio facial, escoliosis, calcificación punteada del cartílago, extremidades proximales y falanges cortas)..$^{10,11,32}$

Una ventaja del uso de heparina durante el embarazo es que no atraviesa la barrera útero-placentaria, en parte debido a que el peso molecular de la HNF es de 40,000 Daltons y de 4,000 a 5,000 Daltons el de la heparina de bajo peso molecular (HBPM). Hemorragia, trombocitopenia y ostopenia son complicaciones que pueden ocurrir en la mujer embarazada con el uso de heparina..$^{10,11,31,32}$

La terapia anticoagulante con HNF es el tratamiento de elección inicial en la mujer embarazada con TEP, ya que su efecto terapéutico se elimina más rápido cuando se suspende, sobre todo en aquellos casos en los que se necesita interrumpir el embarazo de manera urgente. ${ }^{33,34}$

El objetivo primario con la anticoagulación en TEP y embarazo es evitar la reembolización. La anticoagulación se puede conseguir rápidamente con una dosis de carga de 100-120 U/kg intravenosa de HNF, seguida por una infusión continua de $1,000 \mathrm{U} / \mathrm{h}$ o de 15 a $20 \mathrm{U} /$ $\mathrm{kg} / \mathrm{h}$ para mantener el tiempo de tromboplastina parcial activada entre 1.5 y 2 veces el nivel normal. ${ }^{31,34}$

En pacientes embarazadas con TEP que son tratadas con HBPM, se recomienda cambiar a HNF en la semana 36-37 de gestación. Lo anterior tiene como finalidad el uso de una heparina con vida media más corta y, así, evitar mayor riesgo de sangrado durante el parto, pero también ante la eventual necesidad de requerir un procedimiento anestésico regional. La técnica anestésica más segura cuando se decide la interrupción del embarazo es la vía epidural. Este tipo de anestesia puede ser realizada 12 horas después de la última dosis profiláctica de HBPM o 24 horas después de la dosis completa de ésta. Si se administra HNF, el bloqueo epidural puede realizarse con seguridad 6 horas después de suspender la infusión y con un control normal de tiempo parcial de tromboplastina activada previo al procedimiento..$^{10,11}$ Una vez que ocurre el parto y retirado el catéter peridural, 12 horas después puede iniciarse la profilaxis. La anticoagulación terapéutica en mujeres embarazadas que desarrollaron TEP debe continuarse por seis semanas 


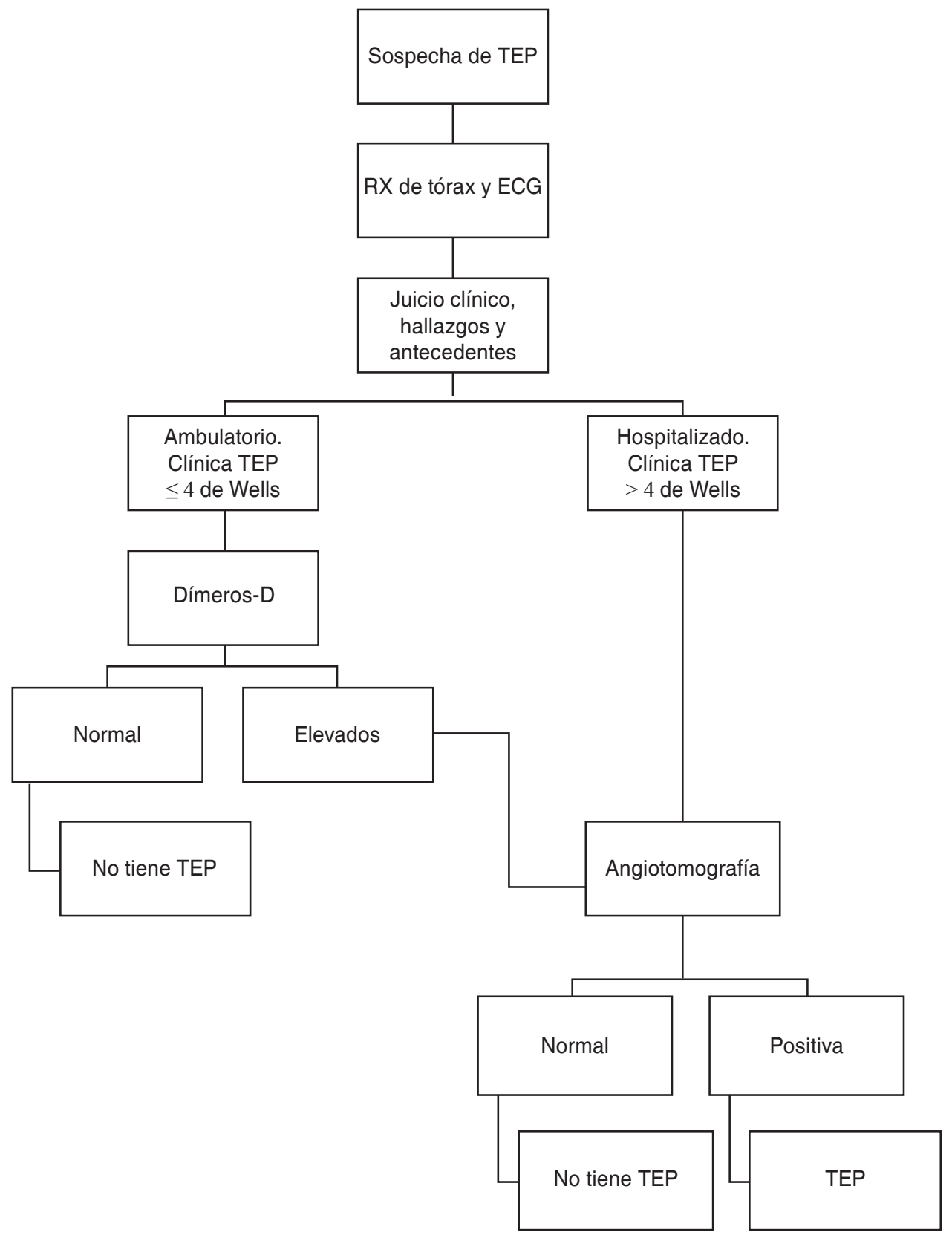

\section{Figura 1.}

Diagnóstico de tromboembolia pulmonar en el embarazo y el puerperio. a seis meses posteriores al parto con HBPM o AVK. Deberá evaluarse el riesgo de trombosis antes de suspender la anticoagulación. ${ }^{10,11,32}$ Cuando por alguna razón debemos revertir los efectos de la heparina, la protamina es el tratamiento de elección. ${ }^{11,31}$

Con la meta de evitar que ocurra un evento de TEP en el período posparto, se recomienda la administración de dosis profiláctica de heparina y el uso de medias de compresión elásticas. Las dosis de HNF y HBPM en el periparto recomendadas por la $\mathrm{ACCP}^{11}$ se señalan en la tabla 2.
Los AVK deberán ser iniciados hasta que el riesgo de hemorragia posparto haya sido superado, siendo al menos dos semanas tras el parto. ${ }^{11}$

La terapia fibrinololítica (TF) está indicada en la población general con TEP aguda y DVD. En la población obstétrica se han reportado casos exitosos con esta terapia con y sin pérdida fetal. ${ }^{24,25}$ La TF está contraindicada en los primeros 10 días posteriores a la operación cesárea, debe reservarse para aquellos casos donde la vida de la madre está en riesgo de muerte inminente, como es el caso de TEP mayor con inestabilidad 
Tabla 2. Anticoagulación periparto con heparina.

HBPM

- Cambiar a HNF en la semana 36-37.

- Suspender al menos $12-24 \mathrm{~h}$ previas a un procedimiento de anestesia regional.

HNF

- Suspender entre 6-24 h previas al parto.

- En caso necesario, revertir la HNF con protamina.

- Reiniciar hasta 12 h después del parto o del retiro del catéter epidural, y 24 h después de la cesárea.

Modificado de: Bates SM, Greer IA, Middeldorp S, et al. Chest 2012;141(Suppl): e691S-e736S.

Abreviaturas: HBPM: Heparina de bajo peso molecular; HNF: Heparina no fraccionada.

hemodinámica y DVD. ${ }^{31}$ La embolectomía percutánea, así como la fragmentación y aspiración del trombo son una opción terapéutica en casos donde el tratamiento convencional ha fallado; su limitante es que dependen de la existencia de un centro con el equipo y personal capacitado para realizar este procedimiento. ${ }^{26,31} \mathrm{La}$ colocación de filtros recuperables en la vena cava inferior es una alternativa cuando la anticoagulación está contraindicada. ${ }^{29,30,35}$

\section{CONCLUSIÓN}

La TEP continúa siendo una de las principales causas de muerte en mujeres durante el embarazo y puerperio. El diagnóstico de TEP en esta población requiere de una alta sospecha clínica, pues puede pasar desapercibido por lo versátil de sus manifestaciones clínicas o quedar enmascaradas por los cambios fisiológicos propios del embarazo. La prueba más útil para el diagnóstico de TEP durante la gestación es la ATAH. El tratamiento está dirigido a lograr una anticoagulación plena. La HNF y la HBPM son los tratamientos de elección debido a que tienen una menor incidencia de complicaciones, un perfil farmacológico más seguro y, por su gran peso molecular, no atraviesan la barrera útero-placentaria. El grupo de mujeres embarazadas con TEP carece de estudios clínicos que permitan hacer un juicio basado en hechos. Es necesaria una mayor investigación en esta área, pues la mayoría de los estudios de TEP excluye a las mujeres embarazadas para su análisis.

\section{REFERENCIAS}

1. Angulo-Vázquez J, Cortés Sanabria L, Torres-Gómez LG, Aguayo Alcázar G, Hernández Higareda S, Ávalos Nuño J. Analysis of maternal mortality during three periods at Hospital de Ginecología y Obstetricia del Centro Médico Nacional de Occidente. Ginecol Obstet Mex 2007;75:384-393.
2. Khan KS, Wojdyla D, Say L, Gülmezoqlu AM, Van Look PF. WHO analysis of causes of maternal death: a systematic review. Lancet 2006;367:1066-1074.

3. Stone SE, Morris TA. Pulmonary embolism during and after pregnancy. Crit Care Med 2005;33:S294-S300.

4. Refuerzo JS, Hechtman JL, Redman ME, Whitty JE. Venous thromboembolism during pregnancy. Clinical suspicion warrants evaluation. J Reprod Med 2003; 48:767-770.

5. Angulo-Vázquez J, Ramírez-García A, Torres-Gómez LG, Vargas González A, Cortes Sanabria L. Pulmonary thromboembolism in obstetrics. Ginecol Obstet Mex 2004;72:400-406.

6. Weiss BM, Hess OM. Pulmonary vascular disease and pregnancy: current controversies, management strategies, and perspectives. Eur Heart J 2000;21:104-115.

7. Hunter S, Robson SC. Adaptation of the maternal heart in pregnancy. Br Heart J 1992;68:540-543.

8. Bourjeily G, Paidas M, Khalil H, Rosene-Montella K, Rodger M. Pulmonary embolism in pregnancy. Lancet 2010;375:500-512.

9. Chan WS, Ray JG, Murray S, Coady GE, Coates G, Ginsberg JS. Suspected pulmonary embolism in pregnancy: clinical presentation, results of lung scanning, and subsequent maternal and pediatric outcomes. Arch Intern Med 2002;162:1170-1175.

10. Santos-Martínez LE, Murillo C, Rodríguez A, et al. Medical reflections on a case of pulmonary thromboembolism in pregnancy. Arch Cardiol Mex 2010;80:255-260.

11. Bates SM, Greer IA, Middeldorp S, et al. VTE, thrombophilia, antithrombotic therapy, and pregnancy: antithrombotic therapy and prevention of thrombosis. 9th ed: American College of Chest Physicians EvidenceBased Clinical Practice Guidelines. Chest 2012;141 (Suppl): e691S-e736S.

12. Wells PS, Anderson DR, Rodger M, et al. Excluding pulmonary embolism at the bedside without diagnostic imaging: management of patients with suspected pulmonary embolism presenting to the emergency department by using a simple clinical model and D-dimer. Ann Intern Med 2001;135:98-107.

13. Troffater KF, Howell ML, Greenberg CS, Hage ML. Use of the fibrin $D$ - dimer in screening for coagulation 
abnormalities in preeclampsia. Obstet Gynecol 1989; 73:435-439.

14. Verduzco Rodríguez L, González Puebla E, Manffrini Madrid F, López Ariza B. D-dimer indifferent stages of pregnancy toxemia. A pilot study. Ginecol Obstet Mex 1998;66:77-80.

15. Bello RF, Patiño A, Dilena F, et al. Preeclampsia severa: dímero $D$ como factor pronóstico en coagulopatía. Infor Med 2001;3:335-343.

16. To MS, Hunt BJ, Nelson-Piercy C. A negative $D$-dimer does not exclude venous thromboemboembolism (VTE) in pregnancy. J Obstet Gynaecol 2008;28:222-223.

17. Pruszczyk P, Bochowicz A, Torbicki A, et al. Cardiac troponin $T$ monitoring identifies high-risk group of normotensive patients with acute pulmonary embolism. Chest 2003;123:1947-1952.

18. Horwich TB, Patel J, MacLellan R, Fonaraw GC. Cardiac troponin I is associated with impaired hemodynamics, progressive left ventricular dysfunction, and increased mortality rates in advanced heart failure. Circulation 2003;108:833-838.

19. Coma-Canela I, Gamallo C, Martínez Onsurbe PM, Lopez-Sendon J. Acute right ventricular infarction secundary to massive pulmonary embolism. Eur Hear J 1988;9:534-540.

20. Kucher N, Printzen G, Goldhaber SZ. Prognostic role of brain natriuretic peptide in acute pulmonary embolism. Circulation 2003;107:2545-2547.

21. McConnel MV, Solomon SD, Rayan ME, Come PC, Goldhaber SZ, Lee RT. Regional right ventricular dysfuntion detected by echocardiography in acute pulmonary embolism. Am J Cardiol 1996;78:469-473.

22. Goldhaber SZ. Echocardiography in the management of pulmonary embolism. Ann Intern Med 2002;136:691700.

23. Cannon C, Goldhaber SZ. Cardiovascular risk stratification of pulmonary embolism. Am J Cardiol 1996;78:11491151.

24. Varsano S, Smorzik Y. Thrombolytic therapy for acute life-threatening pulmonary thromboembolism in a pregnant woman. Isr Med Assoc J 2008;10:740-741.

25. Leonhardt G, Gaul C, Nietsch HH, Buerke M, Schlewssner E. Thrombolytic therapy in pregnancy. J Thromb Thrombolysis 2006;21:271-276.
26. Sato $\mathrm{T}$, Kobatake $\mathrm{R}$, Yoshioka $\mathrm{R}$, et al. Massive pulmonary thromboembolism in pregnancy rescued using transcatheter thrombectomy. Int Heart J 2007;48:269-276.

27. Stein PD, Fowler SE, Goodman LR; The PIOPED II investigators. Multidetector computed tomography for acute pulmonary embolism. N Engl J Med 2006;354:23172327.

28. Matthews S. Short communication: Imaging pulmonary embolism in pregnancy: what is the most appropriate imaging protocol? Br J Radiol 2006;79:441-444.

29. Stone SE, Morris TA. Pulmonary embolism during and after pregnancy. Crit Care Med 2005;33:S294-S300.

30. Marik PE, Plante LA. Venous thromboembolic disease and pregnancy. N Engl J Med 2008;359:2025-2033.

31. Brown HL, Hiett AK. Deep vein thrombosis and pulmonary embolism in pregnancy: diagnosis, complications and management. Clin Obstet Gynecol 2010;53:345359.

32. Mclintock C, Brighton T, Chunilal S, et al. Recommendations for the diagnosis and treatment of deep venous thrombosis and pulmonary embolism in pregnancy and the postpartum period. Aust N Z J Obstet Gynaecol 2012;52:14-22.

33. Barletta JF, Cooper B, Ohlinger MJ. Adverse drug events associated with disorders of coagulation. Crit Care Med 2010;38:S198-S218.

34. James $\mathrm{AH}$. Thromboembolism in pregnancy: recurrence risks, prevention and management. Curr Opin Obstet Gynecol 2008;20:550-556.

35. Gupta S, Ettles DF, Robinson GJ, Lindow SW. Inferior vena cava filter use in pregnancy: preliminary experience. BJOG 2008;115:785-788.

\section{$\triangle$ Correspondencia:}

Dr. Antonio Gerardo Rojas Sánchez, Jefe del Servicio de Fisiología Pulmonar del Hospital Civil de Guadalajara "Fray Antonio Alcalde". Calle Hospital 278, Colonia El Retiro. C.P. 44280, Guadalajara, Jalisco, México.

Teléfono: (33) 39424400 exts. 42027 y 42026

Correo electrónico: gerarojas69@yahoo.com.mx

Los autores declaran no tener conflictos de interés. 